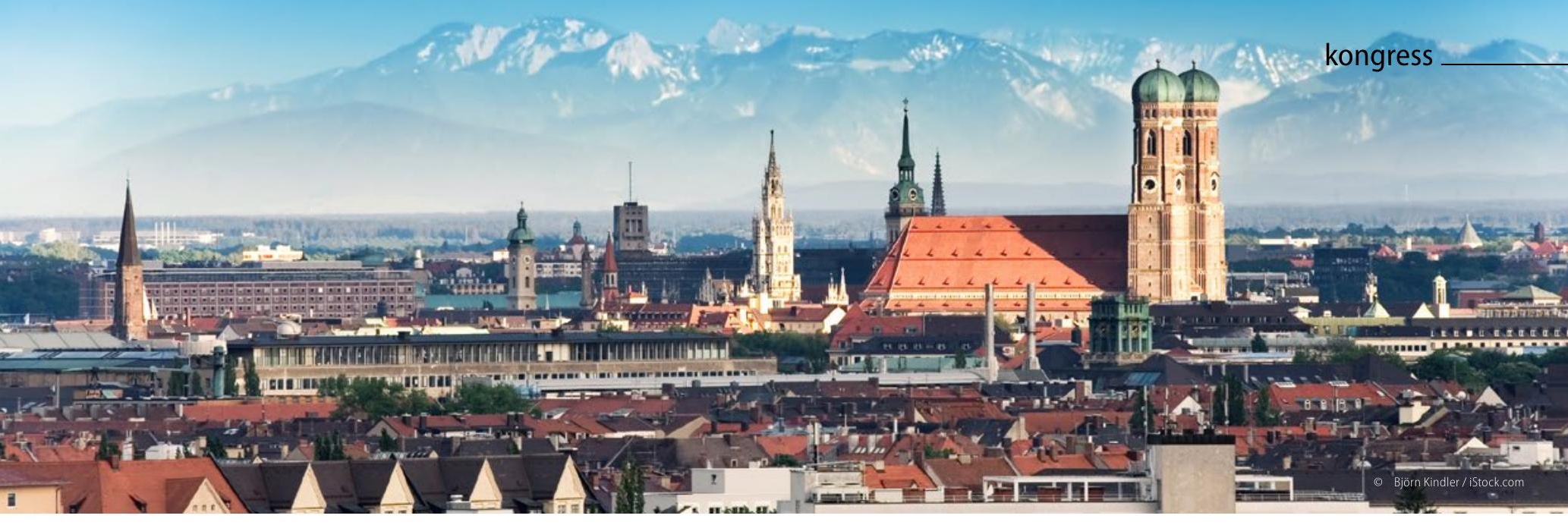

\title{
Aluminium: Keine Chance auf Einhaltung des Grenzwerts
}

Die Weltgesundheitsorganisation (WHO) empfiehlt, die orale Aufnahme von $1 \mathrm{mg}$ Aluminium pro Kilogramm Körpergewicht pro Woche nicht zu übersteigen. Da die Bioverfügbarkeit bei oraler Zufuhr lediglich $0,1 \%$ beträgt, entspricht dies einem Grenzwert von 8,6 Mikrogramm pro Tag bei einem 60 Kilo schweren Menschen.

„Wir wissen, dass die Gesamtaufnahme von Aluminium in Deutschland ganz klar über diesem Wert liegt", sagte Prof. Monika Schäfer-Korting, Institut für Pharmazie der Freien Universität Berlin, auf der 25. Fortbildungswoche für praktische Dermatologie und Venerologie in München.

Ein untersuchter Muffin etwa habe mit $28 \mathrm{mg}$ Aluminium mehr als das Dreifache der empfohlenen Tageshöchstdosis enthalten $(28 \mathrm{mg}$ entsprechen bei 0,1\% Bioverfügbarkeit einer tatsächlichen Aufnahme von 28 Mikrogramm). Neben Süßigkeiten, Käse und Salz sind Backwaren oft am meisten belastet, was an den verwendeten Backblechen liegt, die unter Hitze Aluminium freisetzen. Beim Kochen mit aluminiumhaltigem Geschirr verhält es sich genauso.

Aluminium kommt in Alltagsprodukten in unterschiedlich löslichen Verbindungen vor. In Kombination mit Säure wird das Metall für den menschlichen Organismus leichter resorbierbar - Säure aber ist Bestandteil vieler Lebensmittel und Kosmetika und damit ein Hauptgrund für die Aluminiumanreicherung, etwa in Cremes aus Alu-Tuben. „Auf keinen Fall Zitrusfrüchte oder Aprikosenkuchen in Alufolie einwickeln", nannte SchäferKorting ein weiteres Beispiel.

\section{Vorkommen in Kosmetika}

Die Palette aluminiumhaltiger Kosmetika ist breit: Deodorants, Lippenstifte, Lidschatten und Cremes, aber auch Zahnpasta und Lichtschutzmittel können das Leichtmetall enthalten. „So genau wir die Aufnahme aus dem Darm kennen, so wenig wissen wir über die dermale Resorption von Aluminium Bescheid, hier besteht erheblicher Forschungsbedarf", so Schäfer-Korting. Daher sei die Aufnahme über die Haut mit unwägbaren Risiken verbunden.

Das Bundesinstitut für Risikobewertung (BfR) schätzt, dass bei der Anwendung aluminiumhaltiger Antitranspiranzien etwa 10,5 Mikrogramm Aluminium am Tag in den Körper geschleust werden, deutlich mehr als der WHO-Grenzwert gebietet. Und dieser Wert gilt auch nur für gesunde Haut - schon feinste Verletzungen, die oft nicht bemerkbar sind, etwa nach einer Rasur, erhöhen die Aufnahme um ein Vielfaches.
Das könnte besonders für Frauen von Bedeutung sein. Britische Forscher der Universität Reading haben in Ex-vivo-Versuchen an Brustkrebszelllinien herausgefunden, dass Aluminium das Brustkrebs-unterdrückende Gen und somit die körpereigene DNA-Reparatur hemmt [1, 2]. „Die Induktion von Brustkrebs durch Aluminium halte ich für möglich", positionierte sich Schäfer-Korting.

\section{Aluminium in Impfstoffen}

Viele Impfstoffe enthalten Aluminiumhydroxid zur Bindung der Impfantigene, um eine verzögerte Freisetzung zu erreichen (Wirkungsverstärkung). „Es liegen verschiedene Fallbeobachtungen vor, die erhöhte Aluminiumwerte in den entsprechenden Arealen noch lange nach einer Impfung nachgewiesen haben", so Schäfer-Korting.

So haben französische Wissenschaftler bei der Biopsie eines kutanen Pseudolymphoms, einer gutartigen entzündlichen Hauterscheinung, eine Aluminiumkonzentration von $768 \mathrm{Mi}-$ krogramm pro Gramm Gewebe gemessen - und zwar Jahre, nachdem die Patientin an der betreffenden Stelle geimpft worden war [3]. Bei gesunden Kontrollpersonen betrug die Konzentration noch 5,6 beziehungsweise $9,1 \mu \mathrm{g} / \mathrm{g}$.

Der Einsatz aluminiumhaltiger Impfstoffe ist vor allem bei Säuglingen umstritten. Kanadische Forscher etwa haben in einer epidemiologischen Studie beobachtet, dass die Aluminiumexposition durch Impfungen in den ersten beiden Lebensmonaten mit der Häufigkeit korreliert, später Autismus zu entwickeln [4].

\section{Fazit}

„Aluminium ist kein harmloser Bestandteil unserer Umgebung", warnte die Berliner Wissenschaftlerin. Rechnet man die erwähnten Beispiele und übrigen Quellen einer Aluminiumaufnahme hoch, so wird klar, dass der von der WHO empfohlene Grenzwert für die Tagesdosis derzeit keine Chance hat, eingehalten zu werden. Schäfer-Korting forderte daher, den Zusatz von Aluminium in Lebens-, Arzneimitteln und Kosmetika deutlich zu reduzieren.

Joachim Pfitzer

Schäfer-Korting M. Aluminium in Deo, Nahrung, Impfstoffen - wie gefährlich ist es wirklich?

. Farasani A, Darbre PD. J Inorg Biochem 2015;152:186-9

2. Bakir A, Darbre PD. J Inorg Biochem 2015;152:180-5

3. Guillard O et al. J Trace Elem Med Biol 2012;26:291-3

4. Tomljenovic L, Shaw CA. J Inorg Biochem 2011;105:1489-99 